\title{
Circular polarization of circumstellar water masers around S Per
}

\author{
W. Vlemmings ${ }^{1}$, P. J. Diamond ${ }^{2}$, and H. J. van Langevelde ${ }^{3}$
}

1 Sterrewacht Leiden, Postbus 9513, 2300 RA Leiden, The Netherlands

2 Jodrell Bank Observatory, University of Manchester, Macclesfield, Cheshire, SK11 9DL, England, UK

3 Joint Institute for VLBI in Europe, Postbus 2, 7990 AA Dwingeloo, The Netherlands

Received 12 April 2001 / Accepted 22 June 2001

\begin{abstract}
We present the first circular polarization measurements of circumstellar $\mathrm{H}_{2} \mathrm{O}$ masers. Previously the magnetic field in circumstellar envelopes has been estimated using polarization observations of $\mathrm{SiO}$ and $\mathrm{OH}$ masers. $\mathrm{SiO}$ masers are probes of the high temperature and density regime close to the central star. OH masers are found at much lower densities and temperatures, generally much further out in the circumstellar envelope. The detection of the circular polarization of the $\left(6_{16}-5_{23}\right)$ rotational transition of the $\mathrm{H}_{2} \mathrm{O}$ maser could be attributed to Zeeman splitting due to the magnetic field in the intermediate temperature and density regime. The fields inferred here agree well with predicted values for a combination of the $r^{-2}$ dependence of a solar-type magnetic field, and the coupling of the field to the high density masing regions. We also discuss the unexpected narrowing of the circular polarization spectrum.
\end{abstract}

Key words. masers - polarization - stars: circumstellar matter - stars: individual (S Per) - stars: magnetic fields - stars: supergiants - techniques: interferometric

\section{Introduction}

High mass loss in late type stars produces a circumstellar envelope (CSE) in which several different maser species can be found. These masers, especially $\mathrm{SiO}, \mathrm{H}_{2} \mathrm{O}$ and $\mathrm{OH}$, are excellent tracers of the dynamics and kinematics of the CSEs. Polarization observations of these masers have revealed the strength and structure of the magnetic field throughout the CSE. Observations of $\mathrm{SiO}$ maser polarization have shown highly ordered magnetic fields close to the central star, at radii of 5-10 AU where the $\mathrm{SiO}$ maser emission occurs (Kemball \& Diamond 1997). The standard Zeeman interpretation gives magnetic field strengths of $\approx 5-10 \mathrm{G}$. However, a non-Zeeman interpretation has been proposed by Wiebe \& Watson (1998), which only requires fields of $\approx 30 \mathrm{mG}$. At much lower densities and temperatures and generally much further from the star, $\mathrm{OH}$ maser observations measure fields of $\approx 1 \mathrm{mG}$ (Szymczak \& Cohen 1997; Masheder et al. 1999). But for the intermediate region, at distances of a few hundred $\mathrm{AU}$, no information is available. In this region the $\mathrm{H}_{2} \mathrm{O}$ maser emission occurs. Since water is a non-paramagnetic molecule, determination of the magnetic field is significantly more difficult. The Zeeman splitting of $\mathrm{H}_{2} \mathrm{O}$ is extremely small for the field strengths expected (few hundred $\mathrm{mG}$ ), only $\approx 10^{-3}$ times the typical Gaussian line width of the $\mathrm{H}_{2} \mathrm{O}$

Send offprint requests to: W. Vlemmings,

e-mail: vlemming@strw.leidenuniv.nl maser line $\left(\Delta \nu_{\mathrm{L}} \approx 20 \mathrm{kHz}\right)$. However, Fiebig \& Güsten (1989, hereafter FG) showed that in the presence of such magnetic fields the Zeeman splitting can be detected with high spectral resolution polarization observations. Their observations targeted strong interstellar $\mathrm{H}_{2} \mathrm{O}$ maser features. The observations presented here give the first results of circular polarization measurements of the $\mathrm{H}_{2} \mathrm{O}$ masers found in CSEs. We have used a method similar to that used in FG to determine the magnetic field strength parallel to the line of sight. Like FG we are limiting ourselves to a Zeeman interpretation of the observed splitting, although a non-Zeeman interpretation has been presented in Neduloha \& Watson (1990).

\section{Observations}

The observations were performed at the $\mathrm{NRAO}^{1}$ Very Long baseline Array (VLBA) in December 1998. We observed 4 late type stars (S Per, U Her, VY CMa and NML Cyg), the results presented here are the first results for the supergiant S Per, the source with strong water maser features and relatively simple structure. The stellar velocity $V_{\mathrm{LSR}}$ of S Per is $-38.5 \mathrm{~km} \mathrm{~s}^{-1}$. The beam width at $22.235 \mathrm{GHz}$, the frequency of the $6_{16}-5_{23}$ rotational transition of $\mathrm{H}_{2} \mathrm{O}$, is $\approx 0.7 \times 0.3$ mas. This allows

\footnotetext{
1 The National Radio Astronomy Observatory is a facility of the National Science Foundation operated under cooperative agreement by Associated Universities, Inc.
} 
us to resolve the different $\mathrm{H}_{2} \mathrm{O}$ maser features in the CSE. The data were correlated twice, once with modest $\left(7.8 \mathrm{kHz}=0.1 \mathrm{~km} \mathrm{~s}^{-1}\right)$ spectral resolution, which enabled us to generate all 4 polarization combinations (RR, LL, $\mathrm{RL}$ and LR). The second correlator run was performed with high spectral resolution $\left(1.95 \mathrm{kHz}=0.027 \mathrm{~km} \mathrm{~s}^{-1}\right)$, necessary to detect the circular polarization signature of the $\mathrm{H}_{2} \mathrm{O}$ Zeeman splitting, and therefore only contained the two polarization combinations RR and LL. The data produced by the first correlator run were used to accurately calibrate the $\mathrm{R}$ - and L-polarization. The calibration solutions were obtained on $\mathrm{R}$ and applied to $\mathrm{L}$ after we determined the relative $\mathrm{R}-\mathrm{L}$ corrections, assuming the $\mathrm{R}$ and L-polarization line profiles to be similar. The solutions were then applied to the data-set produced by the second correlator run, which we used to determine the circular polarization $V$ for the separate maser features. The data analysis followed the method of Kemball et al. (1995).

\section{Background}

The $\mathrm{H}_{2} \mathrm{O}\left(6_{16}-5_{23}\right)$ rotational transition consists of 6 hyperfine components. Analyses of interstellar water masers indicate that all 6 hyperfine components contribute to the maser (Walker 1984). The observed intensity ratios however, frequently deviate from those obtained from molecular transition probabilities (Moran et al. 1973; Genzel et al. 1979). Our analysis is performed using fitted line ratios for the 3 strongest hyperfine components $(F=$ $7-6,6-5$ and $5-4)$. The separation between these components is 0.45 and $0.58 \mathrm{~km} \mathrm{~s}^{-1}$ respectively. The weakest components $(F=5-6,5-5$ and $6-6)$ are separated by more than $2.5 \mathrm{~km} \mathrm{~s}^{-1}$. They are not observed in the total power spectrum so we have not included them in our analysis. Our treatment follows closely the analysis performed in FG. Here we have added the possibility of multiple masering hyperfine components. We assume the strongest hyperfine component $(F=7-6)$ to be the dominant transition. Because of the non-paramagnetic nature of the $\mathrm{H}_{2} \mathrm{O}$ molecule, the Zeeman splitting is extremely small. It is due to the interaction of the nuclear magnetic moment with the external $B$ field. Thus the splitting is a factor of $10^{3}$ weaker than that for radicals like OH. In the weak field limit, the split energy $\Delta E_{\mathrm{Z}}$ of a given energy level $(J, F, I)$ is determined by:

$\Delta E_{\mathrm{Z}}=-\left\{\alpha_{J} g_{J}+\alpha_{I} g_{I}\right\} \cdot \mu_{\mathrm{N}} M_{F} \cdot B_{\text {Gauss }}$

with:

$\alpha_{J}=\{J(J+1)+F(F+1)-I(I+1)\} / 2 F(F+1)$,

$\alpha_{I}=\{F(F+1)+I(I+1)-J(J+1)\} / 2 F(F+1)$.

This corresponds to a characteristic frequency shift of the order of $\Delta \nu_{\mathrm{Z}} \approx 10^{3} \mathrm{~Hz} \cdot\left[B_{\text {Gauss }}\right]$. Where $I(=1)$ is the nuclear spin, $\mu_{\mathrm{N}}$ the nuclear magneton and $M_{F}$ the magnetic quantum number; $g_{I}=5.585$, and the $g_{J}$-factors $g_{6}=0.6565$ and $g_{5}=0.6959$ are from Kukolich (1969).

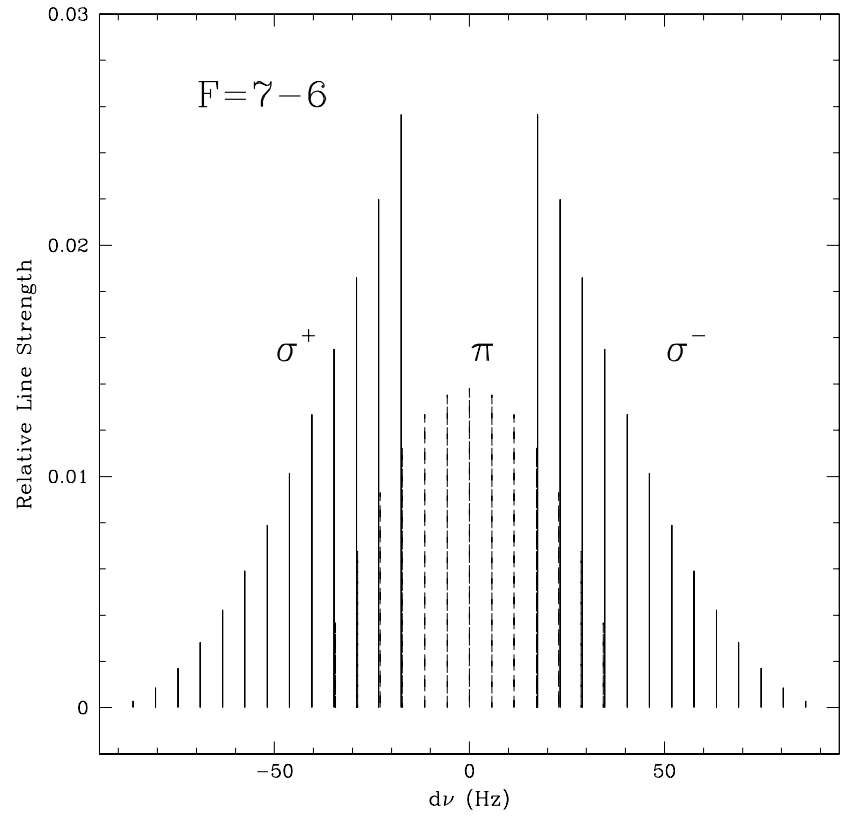

Fig. 1. The Zeeman pattern of the $F=7-6$ hyperfine component for an external field of $50 \mathrm{mG}$.

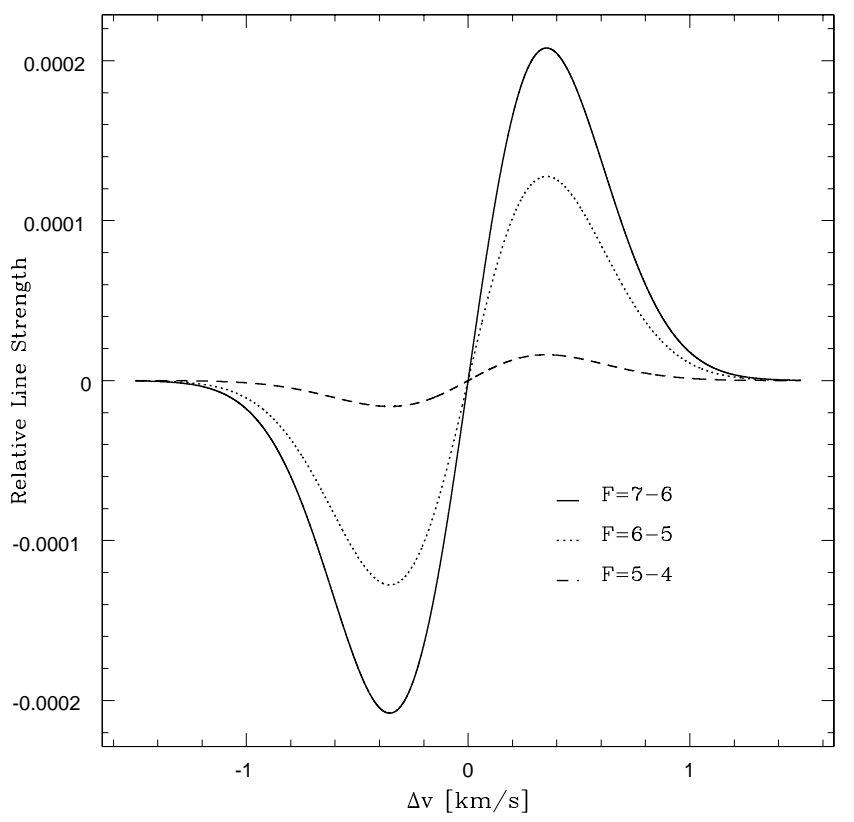

Fig. 2. Synthetic $V$-spectra for $F=7-6,6-5$ and $5-4$ calculated for an external field of $50 \mathrm{mG}$ and a line width of $\Delta v_{\mathrm{L}}=0.5 \mathrm{~km} \mathrm{~s}^{-1}$.

Each hyperfine component will split into 3 groups of lines $\left(\sigma^{+}, \sigma^{-}\right.$and $\left.\pi\right)$, as seen in Fig. 1 for one of the hyperfine components. The relative strengths of the transition probabilities have been determined by Deguchi \& Watson (1986). For a magnetic field $B$ parallel to the line of sight the Zeeman pattern consists of the two circular polarized $\sigma$ components only. The right- and left-handed (RR and LL) spectra, corresponding to the $\sigma^{ \pm}$components will only be slightly shifted against each other $\left(\Delta v_{\mathrm{Z}} \approx 10^{-3}\right.$ to -4 times $\left.\Delta v_{\mathrm{L}}\right)$. As a result, the observed $V$-spectrum (RRLL) will be a sine-shaped function, corresponding to the 
derivative $I^{\prime}$ of the total power spectrum. The amplitude of this function depends on the maser line width, the magnetic field strength, and on which hyperfine components actually contribute to the maser. By calculating synthetic $V$-spectra from the Zeeman pattern for different line widths, magnetic field strength and hyperfine combinations we find the following relation for the percentage of circular polarization:

$P_{V} \equiv\left(V_{\max }-V_{\min }\right) / I_{\max }=A_{\mathrm{F}-\mathrm{F}^{\prime}} \cdot B_{\mathrm{Gauss}} / \Delta v_{\mathrm{L}}$.

Here $V_{\max }$ and $V_{\min }$ are the maximum and minimum of the synthetic $V$-spectrum fitted to the observations. $\Delta v_{\mathrm{L}}\left[\mathrm{kms}^{-1}\right]$ is the Gaussian line width of the total power spectrum, and $I_{\max }$ is the peak flux of the maser feature. $B$ is the magnetic field component along the line of sight. The $A_{F-F^{\prime}}\left[\times 10^{-3}\right]$ coefficient depends on the masering hyperfine components. The $A_{F-F^{\prime}}$ coefficients have been determined by calculating $P_{V}$ from synthetic $V$-spectra, determined for a series of magnetic field strengths $B$, and for the different hyperfine components. For the $F=7-6,6-5$ and $5-4$ components individually we find $A_{F-F^{\prime}}=16.22,10.00$ and 1.23 respectively. For a fitted combination of hyperfine components we find slightly different values. An example of synthetic $V$-spectra for the three hyperfine lines is shown in Fig. 2.

However, due to the complex interactions between the various hyperfine components in the maser regime, deviations from the $V$-spectrum proportionality are possible. Detailed radiative transfer treatment, as performed by Nedoluha \& Watson (1992; hereafter NW), for instance, resulted in $A_{F-F^{\prime}}=23.9$.

\section{Results}

Figure 3 shows the total intensity map of the water maser features surrounding S Per. We are able to identify most of the maser features detected in earlier observations (Diamond et al. 1987; Marvel 1997). The positions are relative to the brightest maser feature, for which we have managed to determine the circular polarization spectrum shown in Fig. 4. This figure also shows a $\chi^{2}$-fit to the sine-shape spectrum. The amplitude of the $V$-spectrum is only a small fraction $(\approx 1 \%)$ of the total power, so we have only been able to determine the Zeeman splitting, and thus magnetic field strength, for the brightest maser feature. We do not detect circular polarization in any of the other bright maser features although, if it was present at the same absolute level as in the brightest feature, we would have detected it. This further confirms our detection, because a scaled down version of the total power $I$ would also have been detectable on the other strong features if calibration errors were significant.

The total power spectrum indicates that one of the hyperfine components clearly dominates, since the splitting of the hyperfine lines should otherwise have been observable. We have performed a fit to the total power spectrum to determine the maser line width and the best fitted ratio for the three strongest hyperfine transitions. Using this

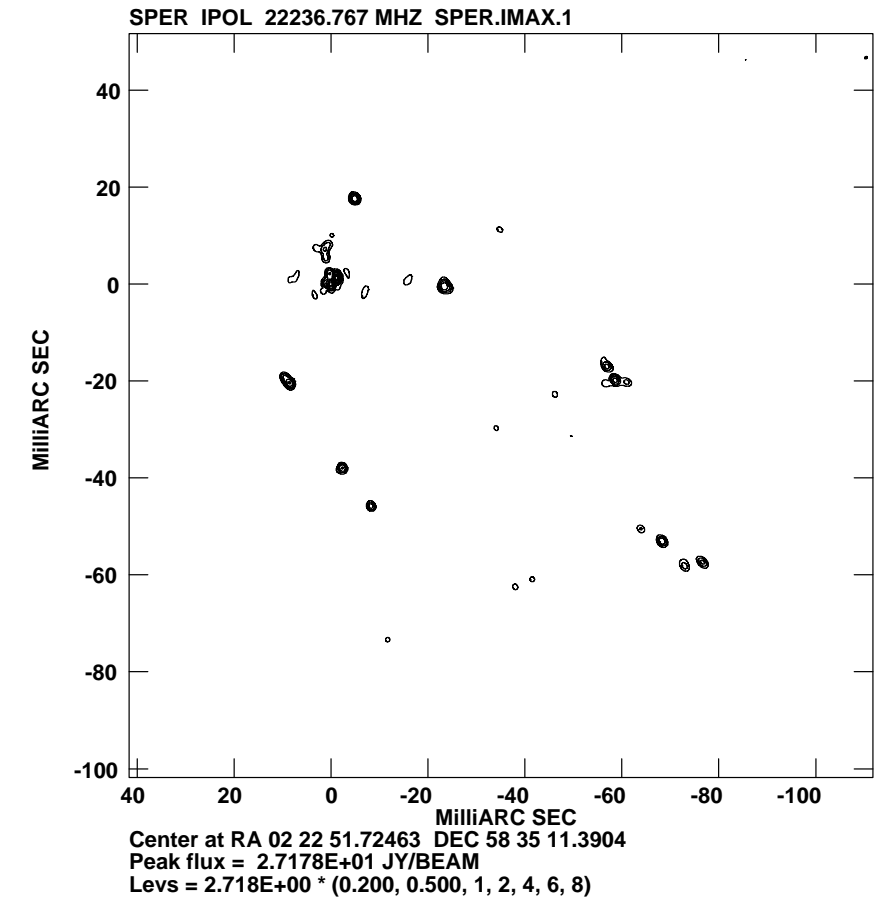

Fig. 3. Total intensity image of the $\mathrm{H}_{2} \mathrm{O}$ maser features around S Per.

line ratio we have calculated the $A_{F-F^{\prime}}$ coefficient as described above. In this case we find $A_{F-F^{\prime}}=15.54$. Using the fitted Gaussian line width $\left(\Delta v_{\mathrm{L}}=0.44 \pm 0.01 \mathrm{~km} \mathrm{~s}^{-1}\right)$ and $P_{V}=(9.9 \pm 0.5) \times 10^{-3}$ in Eq. (2), we find for the magnetic field strength along the line of sight $B_{\|}=$ $279 \pm 30 \mathrm{mG}$. If only the $F=7-6,6-5$ or $5-4$ hyperfine transition contributes the magnetic field should be scaled by $0.96,1.55$ or 12.63 respectively. As seen in Fig. 4 the $V$-spectrum is negative on the blue shifted side, therefore the observed component $B_{\|}$is pointing away from us. This result is the first measured circular polarization in the circumstellar water maser region.

\section{Discussion}

As discussed above, the magnetic field strength was obtained by using a best fitted line ratio for the three main hyperfine components. A radiative transfer treatment for the polarized maser radiation of the $6_{16}-5_{23} \mathrm{H}_{2} \mathrm{O}$ rotational transition was performed by NW. Some difficulties exist matching their results to our observations of the total intensity spectrum, which shows an almost Gaussian shape. Their treatment also did not predict the antisymmetric shape of the $V$-spectrum as shown in our observations and those by FG. However, since the calibration performed here and by FG assumes similar R- and L-polarization line profiles, the anti-symmetric shape is a necessary result of the treatment of the data.

The observed $V$-spectrum however, is not directly proportional to $I^{\prime}$ either, as would have been expected in the simple model. The minimum and maximum of the sineshaped function are not located at $\pm \sigma / \sqrt{2}$, with $\sigma$ being the observed Gaussian line width. Instead they are found 


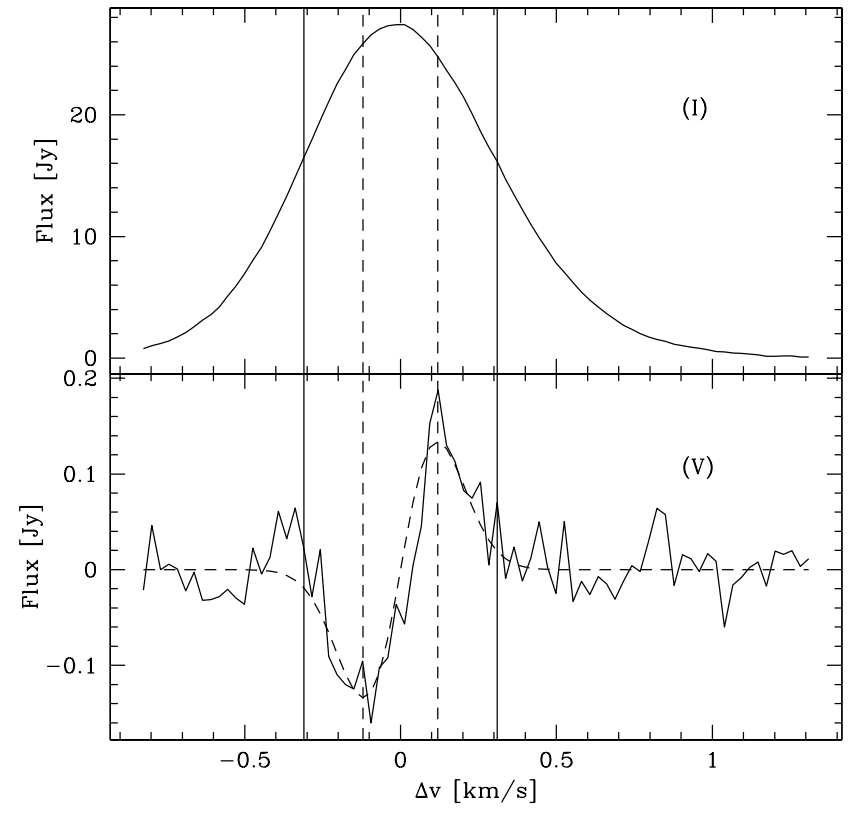

Fig. 4. Total power $(I)$ and circular polarization $(V)$ spectrum of the brightest $\mathrm{H}_{2} \mathrm{O}$ maser feature around $\mathrm{S}$ Per. The dashed line is the fit of the synthetic $V$-spectrum to the observed spectrum. Also shown are the observed (dashed) and expected (solid) positions of the minimum and maximum of the $V$-spectrum.

at $\Delta \nu$ smaller by a factor of $\approx 2.5$ with respect to the line width fitted to the total power spectrum. Possibly, this narrowing of the $V$-spectrum can be attributed to the overlap of the multiple hyperfine components, as predicted by the treatment and analysis of NW. The observed effect, however, still seems too large. Due to this our magnetic field strength could be overestimated by at most a factor of 2. The interstellar water masers observed by FG did not show this narrowing.

Because of the narrowing of the $V$-spectrum it is difficult to address the saturation state of the maser. Elitzur (1998) showed that the observed circular polarization spectrum would be a good indication of the maser saturation state. If the ratio of $V(\nu) / I^{\prime}(\nu)$ increased towards the wings of the line instead of showing a constant ratio the maser is thought to be unsaturated. This is however, almost the opposite effect of what we observe. Until the narrowing of the circular polarization is fully explained our observations are difficult to reconcile with a specific saturation state.

We have also tried to determine the linear polarization of the maser features around S Per, but no indication of linear polarization has been found. This is consistent with the observations of Barvainis \& Deguchi (1989). They explain that the absence of linear polarization is probably due to the fact that masers are not very strongly saturated, and that infrared radiation does not contribute significantly to the pumping process.

The magnetic field strength derived this way is within the range estimated by previous observations of $\mathrm{SiO}$ and $\mathrm{OH}$ masers. As noted before, polarization observations of $\mathrm{SiO}$ masers close to the central star reveal fields of 5-10 G, assuming a standard Zeeman interpretation. $\mathrm{OH}$ maser observations of features around S Per indicate a field of slightly less than $1 \mathrm{mG}$ (Masheder et al. 1999). Based on these values, the dependence of $B \propto r^{-2}$ for a solar-type magnetic field is the most likely. For a dipole medium, the magnetic field is expected to vary with $r^{-3}$, which appears to be too steep to accurately describe the observations.

In $\mathrm{S}$ Per, the $\mathrm{H}_{2} \mathrm{O}$ and $\mathrm{OH}$ masers are observed to exist at similar projected distances (Masheder et al. 1999). This would disagree with the observed differences in the magnetic field strengths, except if the magnetic field could remain frozen in high density clumps. The magnetic field strength is then expected to vary with number density as $B \propto n^{k}$, with $1 / 3 \leq k \leq 1 / 2$ from theoretical predictions (e.g. Mouschovias 1987), where $n$ is the number density. SiO masers are observed in high density clumps at 5-10 AU from the central star. $\mathrm{H}_{2} \mathrm{O}$ masers exist in similar clumps at distances of a few hundred AU, with the magnetic field lines frozen in the dense medium. Richards et al. (1999) show that the $\mathrm{OH}$ and $\mathrm{H}_{2} \mathrm{O}$ maser clumps avoid each other, although located at similar projected distances. They conclude that the density ratio between the $\mathrm{H}_{2} \mathrm{O}$ maser clumps and the $\mathrm{OH}$ in the surrounding medium only needs to be a factor of 50. However, magnetic fields frozen into the maser clumps would require a density ratio of $\approx 10^{4}$ to explain the difference in field. This seems to indicate that actual coexistence between the $\mathrm{OH}$ and $\mathrm{H}_{2} \mathrm{O}$ masers is unlikely.

In conclusion, although the exact influence of the hyperfine interaction is not yet clear, we derive a magnetic field strength of $B_{\|}=279 \pm 30 \mathrm{mG}$.

Acknowledgements. This project is supported by NWO grant 614-21-007.

\section{References}

Barvainis, R., \& Deguchi, S. 1989, AJ, 97, 1089

Deguchi, S., \& Watson, W. D. 1986, ApJ, 302, 750

Diamond, P. J., et al. 1987, A\&A, 174, 95

Elitzur, M. 1998, ApJ, 504, 390

Fiebig, D., \& Güsten, R. 1989, A\&A, 214, 333

Genzel, R., et al. 1979, A\&A, 78, 239

Kemball, A. J., Diamond, P. J., \& Cotton, W. D. 1995, A\&AS, 110,383

Kemball, A. J., \& Diamond, P. J. 1997, ApJ, 481, L111

Kukolich, S. G. 1969, J. Chem. Phys., 50, 3751.

Marvel, K. 1997, Ph.D. Thesis, New Mexico State University Masheder, M. R. W., Van Langevelde, H. J., Richards, A. M. S., Greenhill, L., \& Gray, M. D. 1999, NewAR, 43, 563

Moran, J. M., et al. 1973, ApJ, 185, 535.

Mouschovias, T. Ch 1987, Physical Processes in Interstellar Clouds, ed. G. E. Morfill, \& M. Scholer (Reidel, Dordrecht), 453

Nedoluha, G. E., \& Watson, W. D. 1990, ApJ, 361, L53

Nedoluha, G. E., \& Watson, W. D. 1992, ApJ, 384, 185

Richards, A. M. S., Yates, J. A., \& Cohen, R. J. 1999, MNRAS, 306,954

Szymczak, M., \& Cohen, R. J. 1997, MNRAS, 288, 945

Walker, R. C. 1984, ApJ, 280, 618

Wiebe, D. S., \& Watson, W. D. 1998, ApJ, 503, L71 